\title{
A Target Motion Analysis Algorithm based on Doppler Frequency Shift Optimize Search
}

\author{
Song $\mathrm{Xu}^{1}$, Yang $\mathrm{Ju}^{2, \mathrm{a}}$, Xiaoliang Zhang ${ }^{2, \mathrm{c}}$ \\ ${ }^{1}$ Systems Engineering Research Institute, Beijing, China \\ ${ }^{2}$ Science and Technology on Underwater Acoustic Antagonizing Laboratory, Beijing, China \\ ajyang-pioneer@163.com
}

Keywords: TMA; Doppler Frequency Offset; Multi-peak; Local Optimization.

\begin{abstract}
To solve the problem that the algorithm's slow convergence, or the lower computational efficiency, which caused by the uncertainty of the search accuracy in Doppler frequency shift space, an improved target motion analysis algorithm of bearing-line Doppler frequency shift is introduced. The filter equation is transformed from the nonlinear field to the simple linear form field. When the Doppler frequency is matched, a new optimize search matching algorithm is introduced, and the best estimate problem of the search element's objective function is transformed into a multi-peak and nonlinear optimization problem. Experiments show that the algorithm can decrease the time and space complexity of the algorithm, improve the computational efficiency and convergence precision, and reduce the tracking time.
\end{abstract}

\section{Introduction}

Target motion parameters' calculation is the core content of submarine operational command and weapons control. Researchers propose single array passive bearings-only algorithm[1], nonlinear least squares filtering[2], Kalman filtering[3], unscented Kalman filter[4] and particle filter[5] to solve the target motion problem. The essence of these solutions is to fit the target movement route using the observation from sonars repeatedly. The observatory needs certain motorized movement in order to achieve the location tracking ability of these algorithms. But motor may be dangerous in complex battle environment, and thus brings certain restrictions to the observability and concealment requirement. In order to overcome the above problems, researchers put up a multi-array passive bearings-only algorithm[6, 7]. But it requires multi synchronized stations and data transmission which depend on the communication between stations heavily. In the low-frequency band, the radiated noise is stable and its power and inertia are relatively large, which brings us a new direction to estimate the target motion elements. A Doppler shift based target motion analysis algorithm is proposed in[8], which can estimate the target's motion parameters without pre-extraction of the target's spectrum. But the observation platform needs to standstill.

In this paper, we consider the target's azimuth changing rate and Doppler frequency shift information line under the premise that both the observation platform and the target keep uniform direct movement. Firstly, we perform a filtering process on the azimuth information, and transform the problem into a nonlinear multi peak optimization one using the line spectrum's Doppler shift matching whose best estimation is processed by the locally optimal and search elements' objective function. Finally, we get the information about the target's motion parameters.

\section{Doppler Space Frequency Search Optimization}

The basic idea of space optimization using Doppler shift matching method can be divided into two stages: (1) in the search space, generate the initial elements randomly, each element evenly distributed in the search space, and is independent of each other. Assume that each element is a quality individual; the objective function of the element has an inversely proportional relationship with the quality function. In that way, the reciprocal value of the element's objective function can be 
treated as its quality; (2) the location of the centroid search element is determined according to the various elements of its quality and current location. Scattered elements converge to the centroid position due to the gravitational attraction. At the same time, new search elements will be generated according to certain rules to process Doppler frequency shift optimization matching near the centroid. Doppler frequency search space optimization steps are described as follows.

Step 1: determine the search space $v_{m} \in\left(v_{\operatorname{mim}}, v_{\max }\right), c_{m} \in\left(\hat{c}_{m}-\delta, \hat{c}_{m}+\delta\right)$ according to the least square filter. This search space is two-dimensional which can be evenly cut into rectangular cells. Take the center point between each cell as one search element $P=\left[v_{m}, c_{m}\right]^{T}$.

Step 2: calculate the objective functions of the search elements which are generated in step 1 . When the search element $P$ matches the target's motion parameters, the objective function takes the maximum value, and we can find the location of the best element $P^{\prime}$.

Step 3: calculate a set of cluster centers based on the best element in step 2, determine whether the centers meet the termination condition. If it is yes, jump to step 5, otherwise go to step 4;

Step 4: Determine the new search space and improve the search accuracy based on a set of cluster centers in step $3^{\left\{P_{1}, P_{2}, \cdots, P_{n}\right\}}$, and set the center of cluster centers as new centers.

Step 5: get the target's parameters based on the optimal location of the search elements coupled with the equation mentioned above.

\section{Optimized TMA Algorithm based on The Matching Space}

The process of the target motion analysis algorithm simulation system is shown on the left of Fig. 1. Firstly, process least square filtering among the azimuth sequence and the time sequence which are obtained from the observation platform. Then, estimate the target's azimuth value and determine the initial search space based on the target's speed. Finally, solve the target's motion parameter using Doppler frequency optimization matching search algorithm.

The flow chart of the Doppler frequency optimization matching search algorithm is shown on the right of Fig. 1. This algorithm contains two stages: (1) determine the search space according to the estimated value of azimuth and speed. Divide the search space into several rectangular cells and take each cell's center as an initial candidate solution. (2) Determine whether the centers are stable based on a set of cluster centers in (1). If they are stable, the system can output the target's motion parameters, otherwise generate new search space near the cluster centers and improve the search accuracy, continue the iteration until the centers are stable.
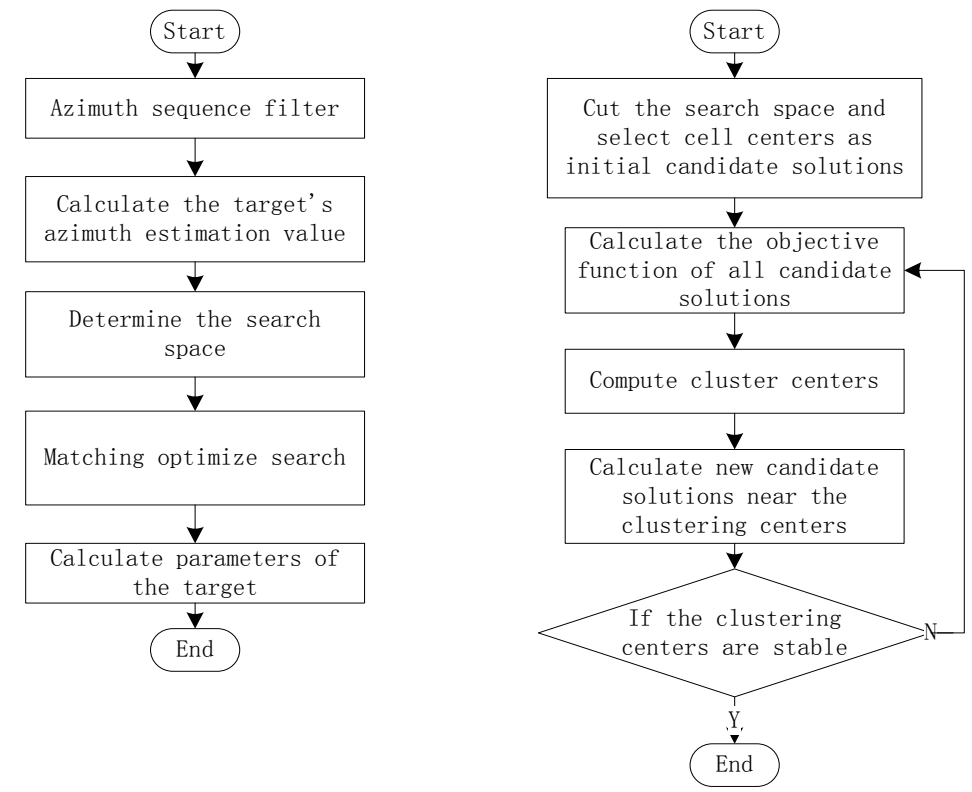

Fig.1 Related Flow charts 


\section{Experiments and discussion}

Assume that the target and the observation platform are both in uniform linear motion. At time $t_{0}\left(t_{0}=0\right)$, Write the distance between the target's position $M_{0}$ and the observation platform's position $W_{0}$ as $D_{0}$, write the initial azimuth as $F_{0}$; at time $t=i$, write the target's position as $M_{i}$, the observation platform's position as $W_{i}$, the azimuth as $F_{i}$, the change of azimuth as $\Delta F_{i}$. Set the target's initial speed as $18 \mathrm{kn}$, course as 120 degrees, and azimuth as 0 ; set the observation platform's speed as $6 \mathrm{kn}$, course as 0 , and set the initial distance between the target and the platform as $20 \mathrm{~km}$.

Using the Matching optimization algorithm to search the search elements, the change of the target's speed and its distance from the observation platform is shown in Fig. 2 and Fig 3. The solid line is the estimated value of the matching optimization algorithm, while the dotted line is the traditional algorithm's estimated value, and the horizontal line in the middle is the real value.

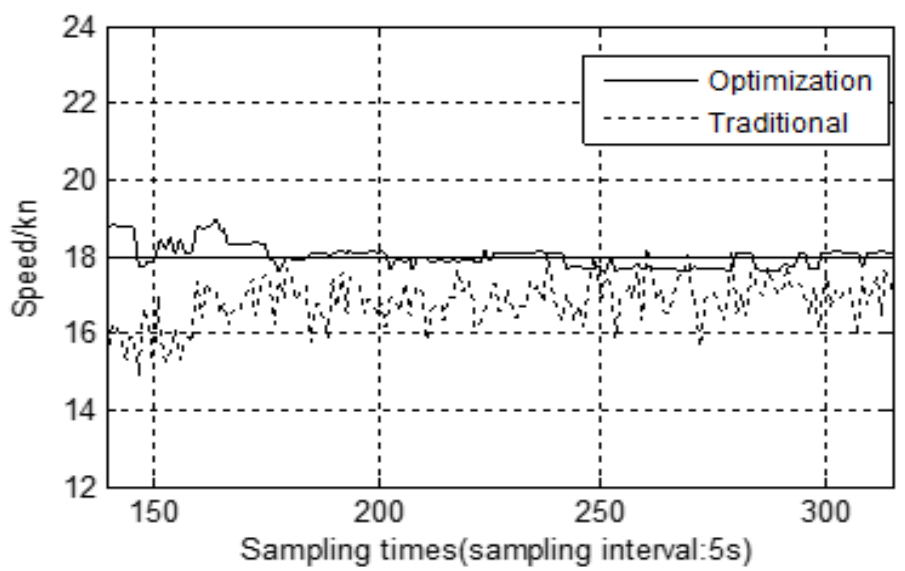

Fig. 2 Changes of the target's speed

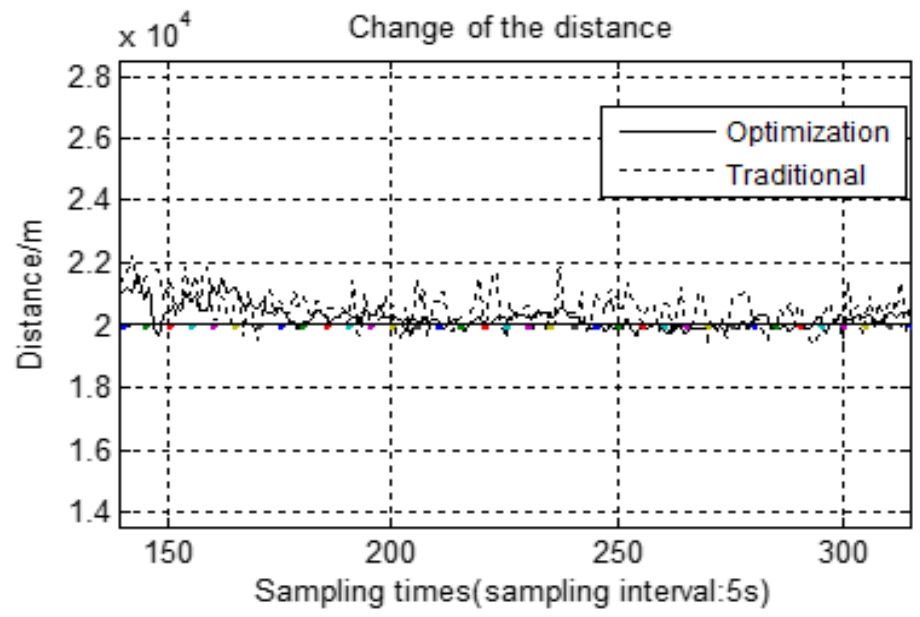

Fig. 3 Changes of the target’s distance

The parameters' error and mean square error are shown in Table 1. It is obvious that parameters solved by optimization algorithm are relatively stable, the convergence time of the matching search algorithm is $725 \mathrm{~s}$, while the traditional algorithm is $760 \mathrm{~s}$. We can conclude that, the convergence speed and convergence accuracy are improved compared with the traditional algorithm.

Table 1: Comparisons of the parameters' error and mean square error

\begin{tabular}{|c|c|c|c|}
\hline & Speed/kn & Azimuth/degree & Distance/m \\
\hline $\begin{array}{c}\text { Traditional } \\
\text { algorithm }\end{array}$ & 1.0538 & 1.1305 & 352.741 \\
\hline Optimized algorithm & 0.4227 & 0.2052 & 166.812 \\
\hline
\end{tabular}




\section{Conclusions}

In this paper, we mapped the filter equations from the nonlinear form to simple linear form using least squares method and target motion analysis algorithm based on the azimuth sequence and the Doppler line spectrum frequency shift information. And we proposed a matching optimization algorithm during the search of the Doppler frequency. The simulation results showed that the new algorithm achieved passive target tracking and positioning, also it reduced the complexity of space-time, improved the efficiency of calculation, and the motion parameters' convergence time and convergence accuracy of the target were also been improved.

\section{References}

[1] Jauffret Claude, Denis Pillon and Annie-Claude Pignol. Bearings-Only Maneuvering Target Motion Analysis from a Nonmaneuvering Platform [J]. IEEE Transactions on Aerospace and Electronic Systems, 2010, 46(4):1934-1948.

[2] ZhiRong Dong. Nonlinear Least-Square Algorithms Used for TMA in Bearing-only System--The Engineering Mathematic Model and Algorithms [J]. Information Command Control System and Simulation Technology,2005,27(2):4-7.

[3] Wang Xing-Bo, Fu Min-Yue, Zhang Huan-Shui. Target tracking in wireless sensor networks based on the combination of KF and MLE using distance measurements [J]. IEEE Transactions on Mobile Computing, 2012, 11(4): 567-576.

[4] Jian Liu, Zhong Liu. Application of UKF in Bearings-only Target Motion Analysis [J]. Journal of Nanjing University of Science And Technology,2008,32(2):222-226.

[5] Chongming Hou, Guanglin Yuan, Jian Wang. A Fish Tracking Algorithm Based on Particle Filtering and Affine Transform [J]. Computer Technology and Development,2014,24 (4):135-138.

[6] Han Shu-Ping, Xu Zhao-Peng. Passive tracking of maneuvering target based on bearings-frequency of two sonar sensors [C]. IEEE International Conference on Electronics, Communications and Control (ICECC), 2011: 2174-2177.

[7] Chen Hui, Han Chong-Zhao, Lian Feng. Three-dimensional target motion analysis using angle-only measurements[C]. IEEE International Conference on Signal Processing, Communication and Computing (ICSPCC), 2013:1 - 6.

[8] Jin Wan, Zhijie Song. A New TMA based on Bearings and Line Doppler Shift [J]. Fire Control and Command Control,2008,33(8):58-61. 